\title{
Research on Fatigue Damage in High-Strength Steel (FV520B) Using Nonlinear Ultrasonic Testing
}

\author{
Bingbing Chen, Chao Wang, Pengfei Wang ${ }^{D}$, Sanlong Zheng, and Weiming Sun \\ Institute of Process Equipment and Control Engineering, Zhejiang University of Technology, Hangzhou 310023, China \\ Correspondence should be addressed to Pengfei Wang; pfwang@zjut.edu.cn
}

Received 22 April 2020; Revised 15 July 2020; Accepted 3 August 2020; Published 19 August 2020

Academic Editor: Yuri S. Karinski

Copyright ( $\odot 2020$ Bingbing Chen et al. This is an open access article distributed under the Creative Commons Attribution License, which permits unrestricted use, distribution, and reproduction in any medium, provided the original work is properly cited.

\begin{abstract}
In view of the early fatigue damage of high-strength steel FV520B, a nonlinear ultrasonic detection was performed on two types of fatigue samples using nonlinear Lamb waves. The experimental results indicated that the ultrasonic nonlinear parameter is highly sensitive to early fatigue damage in high-strength FV520B. For plate specimens, the ultrasonic nonlinear parameter increased with the number of fatigue cycles. Scanning electron microscopy (SEM) observations of the fatigue specimens revealed that as the number of fatigue cycles increased, the microstructure of the material gradually deteriorated, and the ultrasonic nonlinear parameter increased. For notched specimens, the ultrasonic nonlinear parameter increased as the size of the main crack increased. SEM observations of the fracture indicated that the ultrasonic nonlinear parameters were more consistent with the equivalent microcrack length (defined as the sum of microcrack lengths in the statistical area), as compared with the length of the main crack. It was determined that the nonlinear effect of the Lamb wave is related to the equivalent microcrack length inside the material and that the ultrasonic nonlinear parameter can effectively characterize the fatigue damage state of high-strength FV520B.
\end{abstract}

\section{Introduction}

High-strength FV520B is often used to make centrifugal compressor impellers. A centrifugal compressor is an important energy conversion device and is widely used in the petroleum, chemical, metallurgy, natural gas transmission, aero-engine, and mine ventilation industries, and in other important fields [1-3]. The impeller is an integral part of the centrifugal compressor associated with high rotational speeds and complex loading. The impeller often generates small vibrations at high frequencies; thus, it is prone to fatigue failure $[4,5]$. The accidents caused by fatigue failure are often catastrophic and can cause massive losses. Therefore, the study of ultrasonic nondestructive testing technology for the early fatigue damage of high-strength FV520B is of great significance.

For most well-designed engineering components, the early damage of the material (before the formation of macrocracks) accounts for more than $80 \%$ of the component life [6]. While the existing nondestructive testing technology, which includes linear ultrasonic testing technology, is effective for the detection of macrodefects in materials, it is less sensitive to early damage (e.g., dislocation structure and microcracks) to the materials before macrocracks appear in the fatigue process. Recent studies have shown that the degradation of the early mechanical properties of materials can trigger ultrasonic nonlinear effects [7]. Therefore, the degradation can be used to detect the damage degree of materials at an early stage of fatigue. Studies have shown that the nonlinearity caused by microdefects (such as dislocations) is much greater than that caused by lattice anharmonicity. Jhang and Kim [8] conducted nonlinear ultrasonic testing research on SS41 and SS45 medium-carbon steel under tensile and fatigue loading, respectively, and analyzed the high-order harmonics in the process of ultrasonic propagation. It was found that the nonlinear coefficient increased with increases in tensile load and fatigue cycle. Deng and Pei [9] tested the fatigue damage of aluminum plates using a nonlinear Lamb wave method and discovered that the stress wave factor decreased monotonously with increasing fatigue cycles. Walker et al. [10] used a nonlinear 
Rayleigh wave to characterize the damage to an A36 steel specimen caused by a low-cycle tensile fatigue test. The test results showed that, in the early stages of fatigue life, the nonlinear coefficient increased with an increase of cycle number, whereas when the fatigue cycle number was higher than 20, the nonlinear coefficient slowly decreased. Zhang [11] tested (type) 304 austenitic stainless steel under low-cycle fatigue, using nonlinear ultrasound. It was found that during the low-cycle fatigue damage of the 304 stainless steel, the ultrasonic nonlinear parameters showed a trend wherein it first increased and then decreased. In the initial stages of fatigue damage, the increase in the ultrasonic nonlinear parameters is mainly due to the increase of dislocation density in the plane dislocation structure, and the subsequent decrease of the ultrasonic nonlinear parameters may be related to the formation of dislocation walls and dislocation cell structures. Wang et al. [12] conducted nonlinear ultrasonic testing on three groups of KMN high-strength steel fatigue specimens with different cycles, including a vibration fatigue test, bending fatigue test, and tension bending fatigue test. The results showed that the nonlinear parameters initially increased and then decreased with the increase of fatigue cycles. The microfracture characteristics of the KMN specimens were analyzed under different fatigue test types. It was found that with the increase of fatigue cycle number, the microstructures of the KMN specimens gradually deteriorated, and cracks appeared. Dutta et al. [13] conducted ultrasonic nonlinear detection on aluminum and steel specimens, a strong nonlinear effect from the cracked specimen was observed, and the magnitude of harmonic components increased nonlinearly with the increasing amplitude of the input signal. Shen et al. [14-16] used the local interaction simulation approach (LISA) to study the contact-impact clapping phenomena of the wave-crack interactions, based on the penalty method. They found that the higher harmonics were generated during the nonlinear interaction between fundamental waves and fatigue cracks. The nonlinear scattering and mode conversion phenomenon of Lamb waves as they interact with fatigue crack were also studied. Hong et al. [17] established a model for simulating nonlinear characteristics of ultrasonic waves propagating in a fatigued medium based on nonlinear constitutive relations of the medium and verified it with experiments. The simulation results consisted of the experimental results, both of which show that relative acoustic nonlinearity parameter $\beta^{\prime}$ linearly increased with the wave propagation distance due to the material and geometric nonlinearities. Liu et al. [18] simulated the models with different microcrack sizes and found that Lyapunov exponent had a good linear relationship with the size of the crack. Wu et al. $[19,20]$ studied the nonlinear effect of the interaction of Lamb waves and cracks through experiments and simulations. Results showed that the relative nonlinear parameter linearly increased with the crack propagation.

The research indicated that nonlinear ultrasonic nondestructive testing technology can effectively detect early defects in a material and has broad application prospects.

\section{Theoretical Basis of Nonlinear Ultrasonic Testing}

The example of a one-dimensional longitudinal wave is considered to demonstrate the formation of nonlinear ultrasound in a solid. For most types of materials, the relationship between stress and strain is nonlinear [21] and can be described by Hooke's law in small areas as follows:

$$
\sigma=E \varepsilon(1+\beta \varepsilon+\cdots),
$$

where $E$ is the elastic modulus and $\beta$ is the second-order elastic coefficient (also called the nonlinear coefficient).

The equation for the motion of a particle in the solid medium in the $x$-direction is as follows:

$$
\rho \frac{\partial^{2} u}{\partial t^{2}}=\frac{\partial \sigma}{\partial x}
$$

where $\rho$ is the density of the medium, $x$ is the distance in the direction of wave propagation, $t$ is the time, and $u$ is the displacement of the particle at location $x$ in the medium. Using (1) and (2) simultaneously and ignoring the higherorder terms that exceed the second-order term in (1), an equation for particle displacement can be obtained, which indicates the relationship between particle displacement and strain as follows:

$$
\frac{\partial^{2} u}{\partial t^{2}}=c^{2} \frac{\partial^{2} u}{\partial x^{2}}+2 c^{2} \beta \frac{\partial u}{\partial x} \frac{\partial^{2} u}{\partial x^{2}}
$$

where $c$ is the wave velocity in the medium. According to the perturbation theory, we can assume that the displacement $u$ in (3) is determined by the following equation:

$$
u=u_{1}+u_{n 1} \text {, }
$$

where $u_{1}$ is the solution when $\beta=0$ and $u_{n 1}$ is the first-order perturbation solution. Equation (3) can now be written as follows:

$$
\frac{\partial^{2} u_{1}}{\partial t^{2}}+\frac{\partial^{2} u_{n 1}}{\partial t^{2}}=c^{2}\left(\frac{\partial^{2} u_{1}}{\partial x^{2}}+\frac{\partial^{2} u_{n 1}}{\partial x^{2}}\right)\left[1+2 \beta\left(\frac{\partial u_{1}}{\partial x}+\frac{\partial u_{n 1}}{\partial x}\right)\right] .
$$

In this case, the linear solution is set as follows:

$$
u_{1}=A_{1} \cos (k x-\omega t)
$$

where $\omega$ is the frequency, and if $\lambda$ is the wavelength, then $k=\omega / c=2 \pi / \lambda$ is the wave number. The approximate analytical solutions to (3) and (5) can then be obtained by comprehensively applying the multiscale method and the trial solution and neglecting the high-order small quantity in the solution process as follows:

$$
u=u_{1}+u_{n 1}=A_{1} \cos (k x-\omega t)-A_{2} \sin [2(k x-\omega t)],
$$

where $A_{1}$ and $A_{2}$ represent the fundamental frequency and second harmonic amplitudes, respectively, and 


$$
A_{2}=\frac{\beta}{8} A_{1}^{2} k^{2} x
$$

It can be observed from (7) that the second harmonic amplitude $A_{2}$ describing the nonlinear response is related to $\beta$, which implies that $\beta$ can be used as a parameter to describe the nonlinearity of the medium, as shown in the following equation:

$$
\begin{aligned}
& \beta=\frac{8}{k^{2} x} \frac{A_{2}}{A_{1}^{2}}, \\
& \beta \propto \frac{A_{2}}{A_{1}^{2}} .
\end{aligned}
$$

Therefore, when the ultrasonic frequency and propagation distance are fixed, the ultrasonic nonlinear parameters can be calculated by measuring the amplitudes of the fundamental frequency and the second harmonic. What we care about is the change of nonlinear parameter. For convenience, in this experiment, the relative nonlinear parameter $\beta^{\prime}$ is used to replace the change of the ultrasonic nonlinear parameter:

$$
\beta^{\prime}=\frac{A_{2}}{A_{1}^{2}}
$$

In this article, it is also called ultrasonic nonlinear parameter $\beta$.

\section{Experimental Material and Measurement Methods}

3.1. Experimental Material. FV520B steel is an important material for the manufacture of centrifugal compressor impellers, owing to its high strength, high hardness, good wear resistance, and other excellent mechanical properties. It is usually used in the manufacture of core parts in various large machinery and equipment. The main chemical compositions and mechanical properties of FV520B are shown in Tables 1 and 2.

3.2. Fatigue Test Method. The fatigue specimens were designed as plate specimens and notched specimens, with a thickness of $2 \mathrm{~mm}$. The shapes of the plate and notch fatigue specimens are shown in Figures 1 and 2, respectively. The surface of each specimen was mechanically vibration-polished with emery papers to keep the surface consistent before the fatigue test. The tensile fatigue test was carried out on an electromagnetic resonance high-frequency fatigue testing machine, to obtain a fatigue test sample with different cycles. The load waveform was a sine wave, the stress ratio was 0.1 , and the frequency was $120 \mathrm{~Hz}$. There were nine plate-shaped specimens in total. One was left as the original specimen, and the rest were tested on the fatigue testing machine. The cycle times were $5 \times 10^{4}, 1 \times 10^{5}, 5 \times 10^{5}$, $1 \times 10^{6}, 5 \times 10^{6}, 7 \times 10^{6}, 1 \times 10^{7}$, and $2 \times 10^{7}$, respectively, and the loading stress was $400 \mathrm{MPa}$. The finite element software ANSYS was used to simulate the stresses on the notched specimens; the stress concentration factor at the notch was 5.5. The notched specimens were divided into two groups for testing. The two groups were loaded with stresses of $100 \mathrm{MPa}$ and $120 \mathrm{MPa}$, respectively, and the stress concentrations at the specimen notch were about $550 \mathrm{MPa}$ and $660 \mathrm{MPa}$, respectively. The simulation results are shown in Figure 3.

3.3. Nonlinear Ultrasonic Testing. In this experiment, a longitudinal wave incidence method was used to excite an ultrasonic Lamb wave, which is highly sensitive to damage and ease of actuation. The commonly used S1-S2 mode $[22,23]$ was selected. This mode is relatively easy to excite and satisfies group velocity matching and has a high excitation efficiency. It can be appropriately selected from the other Lamb wave modes, as the group speed is high. An ultrasonic Lamb wave measurement of the FV520B specimens was carried out using a RAM-5000 high-energy ultrasonic system, as shown in Figure 4. A schematic diagram of the nonlinear ultrasonic detection system is presented in Figure 5 .

The excitation signal of the nonlinear ultrasonic system was a 20-cycle Hamming-windowed sinusoidal tone-burst signal. The tone-burst signal was excited by a high-energy ultrasound system and was then processed by an attenuator and a low-pass filter before being transmitted to an ultrasonic piezoelectric transducer. The piezoelectric transducer converted the voltage signal into an ultrasonic signal and transmitted it to the specimen. A piezoelectric transducer at the receiving end converted the received ultrasonic signal into a voltage signal. The received signal was processed by a high-pass filter and a preamplifier and was sent to an oscilloscope and computer for data processing and analysis. A narrow-band piezoelectric transducer with a center frequency of $2.25 \mathrm{MHz}$ and a wide-band piezoelectric transducer with a center frequency of $5 \mathrm{MHz}$ were selected as the transmitting and receiving probes, respectively. A $4 \mathrm{MHz}$ high-pass filter was used to filter the received signal. A signal with a frequency of $2.2 \mathrm{MHz}$ was used as the excitation signal, and the incident angle was $27^{\circ}$. The probe and the specimen were coupled using glycerin. The amplitudes of the fundamental and second harmonic Lamb waves were obtained after the signals were processed by a short-time Fourier transform.

\section{Experimental Results}

4.1. Dispersion Curve and Signal Validity Verification. The second harmonic generation efficiency is low owing to the dispersion characteristics of the ultrasonic guided wave. Furthermore, the second harmonic signal was weak and difficult to measure. If the phase velocity of the fundamental frequency Lamb wave mode excited in the specimen was equal to the phase velocity of the double-frequency Lamb wave mode, the second harmonic signal was relatively easy to measure. Therefore, the group velocity and phase velocity dispersion curves of high-strength FV520B specimen were obtained by solving the Rayleigh-Lamb dispersion equation using MATLAB, as shown in Figure 6. According to the 
TABLE 1: Chemical composition of FV520B (wt.\%).

\begin{tabular}{lcccccccccc}
\hline $\mathrm{C}$ & $\mathrm{Si}$ & $\mathrm{Mn}$ & $\mathrm{P}$ & $\mathrm{S}$ & $\mathrm{Ni}$ & $\mathrm{Cr}$ & $\mathrm{Cu}$ & $\mathrm{Nb}$ & $\mathrm{Mo}$ & $\mathrm{Fe}$ \\
\hline$\leq 0.07$ & $\leq 0.07$ & $\leq 1.0$ & $\leq 0.03$ & $\leq 0.03$ & $5.0-6.0$ & $13.2-14.5$ & $1.3-1.8$ & $0.25-0.45$ & $1.3-1.8$ & $\mathrm{Ba1}$ \\
\hline
\end{tabular}

TABLE 2: Mechanical properties of FV520B.

\begin{tabular}{lllll}
\hline Elasticity modulus $E(\mathrm{GPa})$ & Tensile strength $R_{m}(\mathrm{MPa})$ & Yield strength $R_{\mathrm{p} 0.2}(\mathrm{MPa})$ & Vickers hardness HV $\left(\mathrm{kgf} \cdot \mathrm{mm}^{-2}\right)$ & Elongation $\delta(\%)$ \\
\hline 194
\end{tabular}

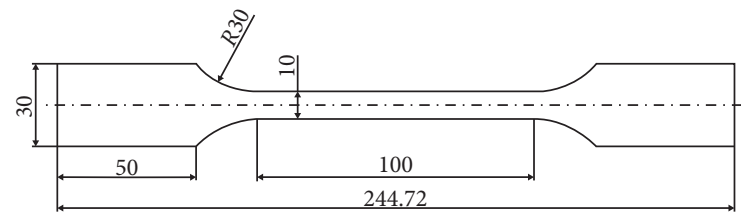

(a)

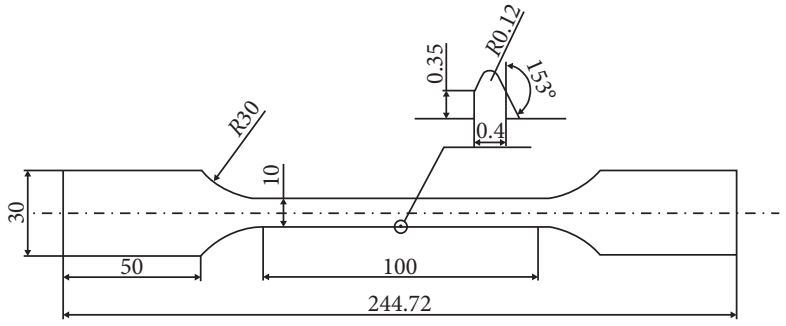

(b)

Figure 1: Fatigue specimen size. (a) Plate specimen. (b) Notched specimen.

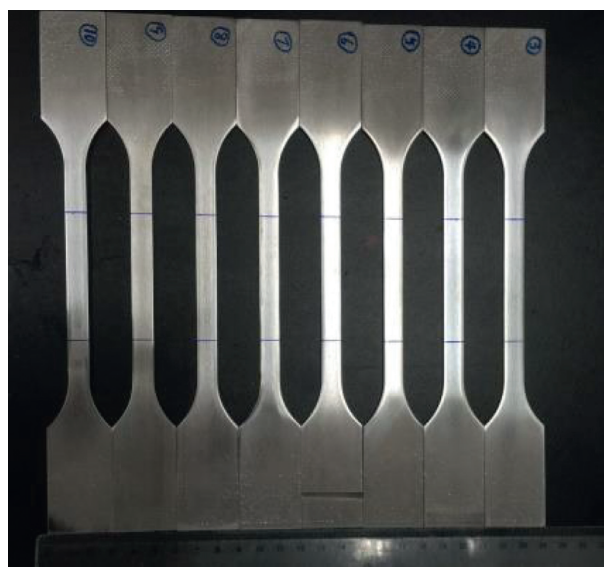

(a)

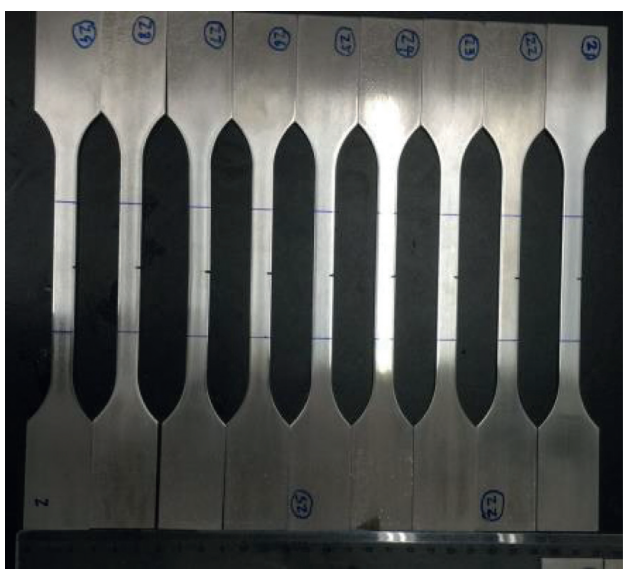

(b)

Figure 2: Photo of fatigue specimen. (a) Plate specimen. (b) Notched specimen.

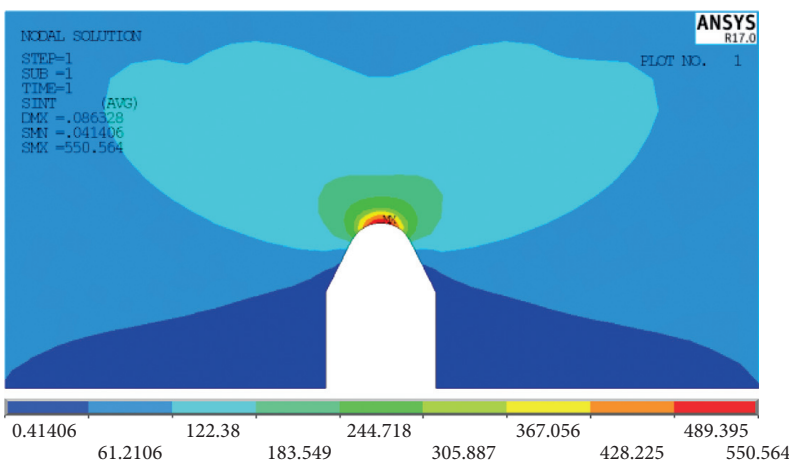

(a)

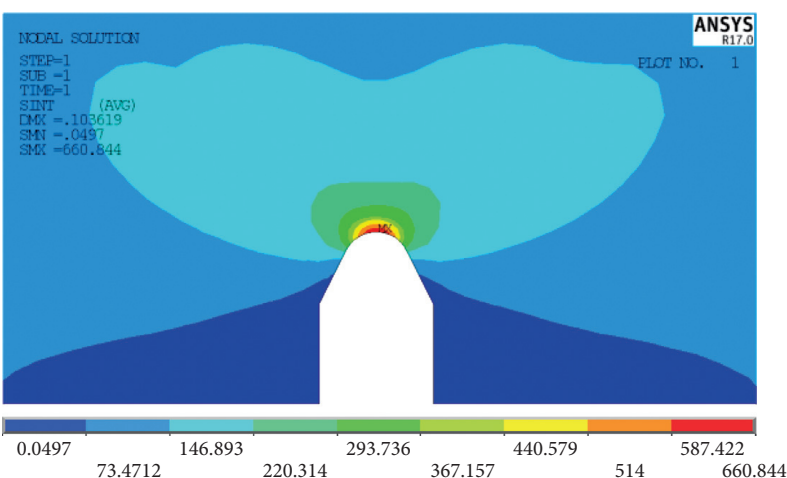

(b)

Figure 3: The results of the finite element simulation at the specimen notch. (a) $550 \mathrm{MPa}$ stress concentration. (b) $660 \mathrm{MPa}$ stress concentration. 


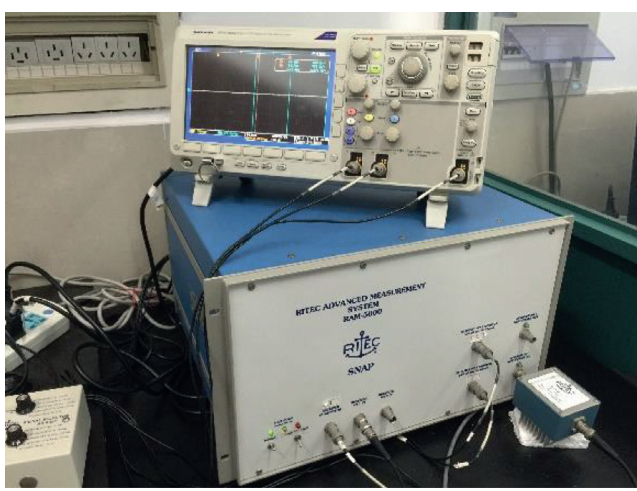

(a)

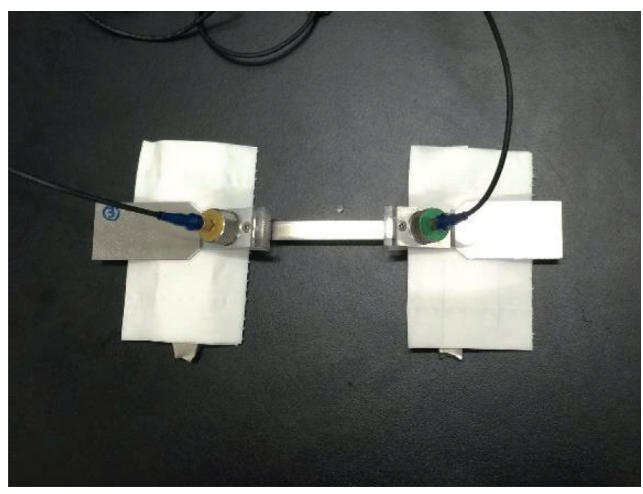

(b)

FIGURE 4: Nonlinear ultrasonic detection system. (a) RAM-5000 high-energy ultrasonic system. (b) Experiment procedure.

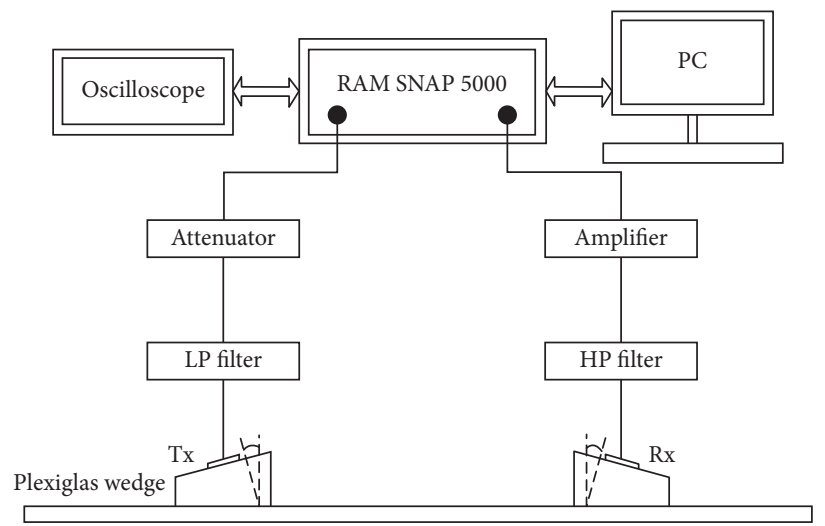

FIgURE 5: The schematic diagram of the nonlinear ultrasonic detection system.

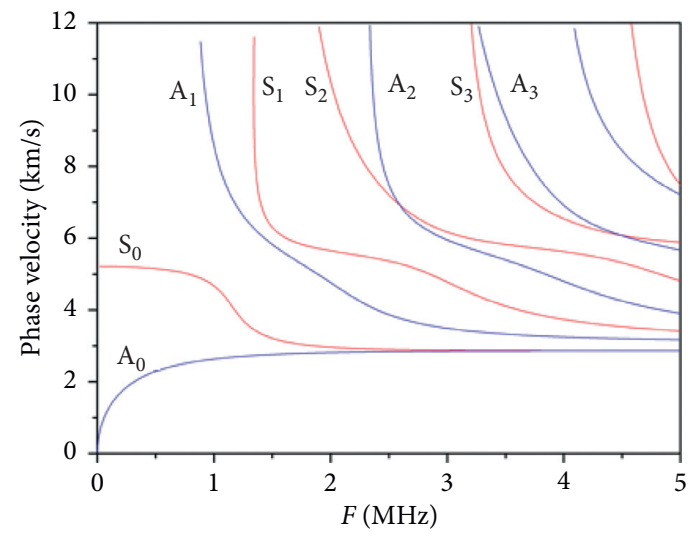

(a)

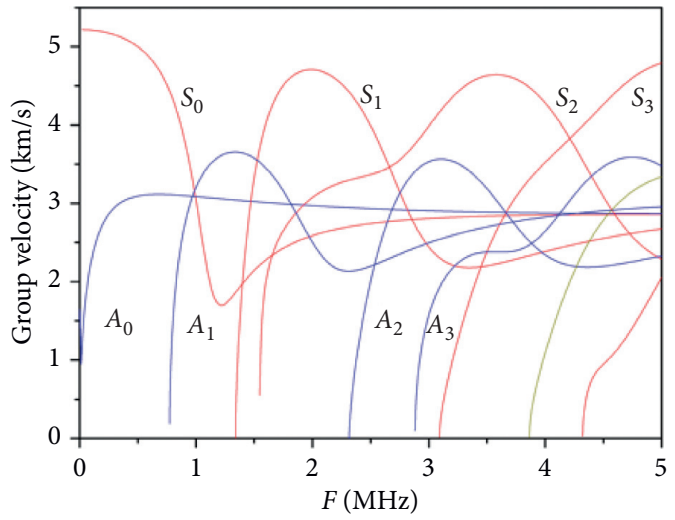

(b)

FIgURE 6: Dispersion curve of the Lamb wave in the FV520B specimen. (a) Phase velocity dispersion curve. (b) Group velocity dispersion curve.

dispersion curve of the phase velocity, the fundamental frequency of the Lamb wave matches the second harmonic phase velocity at $1.83 \mathrm{MHz}$. Due to the interference of experimental equipment, coupling agent, and circuit, the theoretical excitation frequency will shift, resulting in the difference between theoretical calculation and actual experimental measurement. Therefore, frequency sweep of high-strength FV520B specimen was carried out near the theoretical frequency. The amplitude of the second harmonic is the largest when the excitation frequency is $2.2 \mathrm{MHz}$, so the excitation frequency of $2.2 \mathrm{MHz}$ was selected for the nonlinear ultrasound experiment. 
Before the nonlinear ultrasonic Lamb wave measurement of the FV520B specimens, it was necessary to verify the system to ensure that the measured second harmonic signal was caused by the test material rather than by the measurement system. Nonlinear ultrasonic testing was carried out on FV520B fatigue specimens, and the time domain signals were obtained as shown in Figure 7 . The time domain signals were processed with STFT (short-time Fourier transform) and the STFT time-frequency energy spectrum image of FV520B specimen was obtained, as shown in Figure 8. The STFT energy spectrum is represented by 256 levels of gray scale and the deeper the color means the greater the energy, since the amplitude of the fundamental wave and second harmonic wave can be obtained. Then, the values of nonlinear parameter can be calculated. The incident voltage was kept constant, and the distance between the incident transducer and the receiving transducer was changed (from $40 \mathrm{~mm}$ to $80 \mathrm{~mm}$ ). It was found that the nonlinear parameters of the ultrasound showed a linearly increasing trend with the increase of propagation distance, as shown in Figure 9. The results indicate that the second harmonic signal received by the receiving transducer is generated by the fundamental frequency Lamb wave propagation in the specimen, rather than by the system or couplant.

4.2. Experimental Measurement Results. The nonlinear parameter $\beta$ of the fatigue specimens with different fatigue cycles was divided by $\beta_{0}$ ( $\beta_{0}$ is the ultrasonic nonlinear parameter of the original specimen) for normalization, and the normalized nonlinear parameter $\beta$ was obtained. The nonlinear parameters $\beta$ mentioned below are all normalized values. In order to reduce the error caused by nonlinear test, three times of nonlinear ultrasonic testing was carried out for each fatigue specimen in the experiment, and the average value was taken as the test result. The relationship between the normalized nonlinear parameters and fatigue period is used to describe the nonlinear changes of materials owing to fatigue damage, as shown in Figure 10. As seen in Figure 10(a), the nonlinearity parameter showed an increasing trend with an increase of fatigue cycles for the plateshaped specimen (400 MPa loading stress). For the fatigue tests of the notched specimens, the relationship curve also showed a similar trend. Figure 10(b) shows the relationship between the nonlinear parameters and the fatigue cycle of the notched specimen (550 MPa stress concentration). As can be seen from Figure 10(b), the ultrasonic nonlinear parameters increased with the increase of fatigue cycles. However, a significant decrease occurred at point A. Scanning electron microscopy (SEM) observation results indicated that the numbers and sizes of microcracks on the fracture surface of the specimen corresponding to point A were significantly lower than those at other points. An experiment with another group of notched specimens (660 MPa stress concentration) also indicated that the ultrasonic nonlinear parameters increase with the increase of fatigue cycle, as shown in Figure 10(c). The experimental results indicate that the ultrasonic nonlinear parameters are

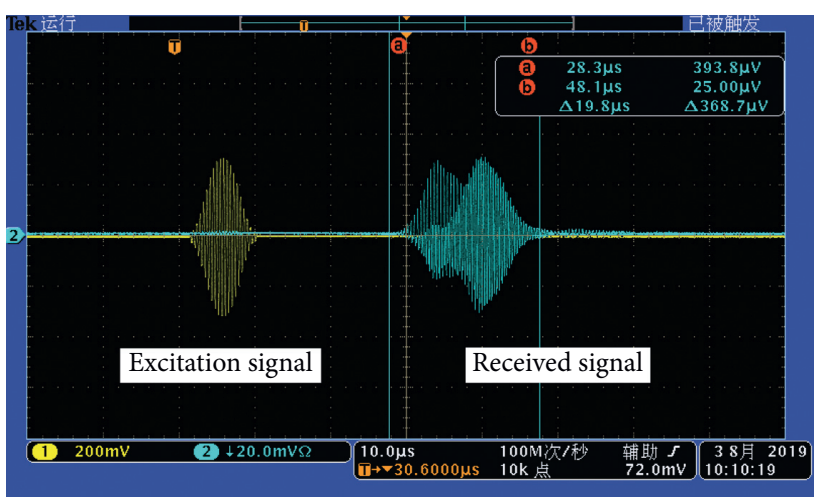

Figure 7: Time domain signals of FV520B specimen (400 MPa loading stress, $N=5 \times 10^{5}$ ).

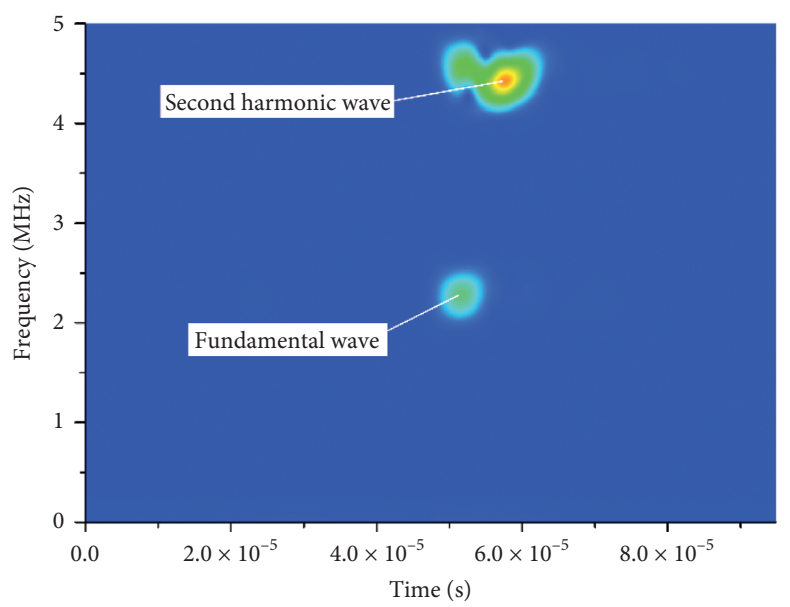

FIgURE 8: STFT spectrograms of FV520B specimen Lamb wave signals (400 MPa loading stress, $N=5 \times 10^{5}$ ).

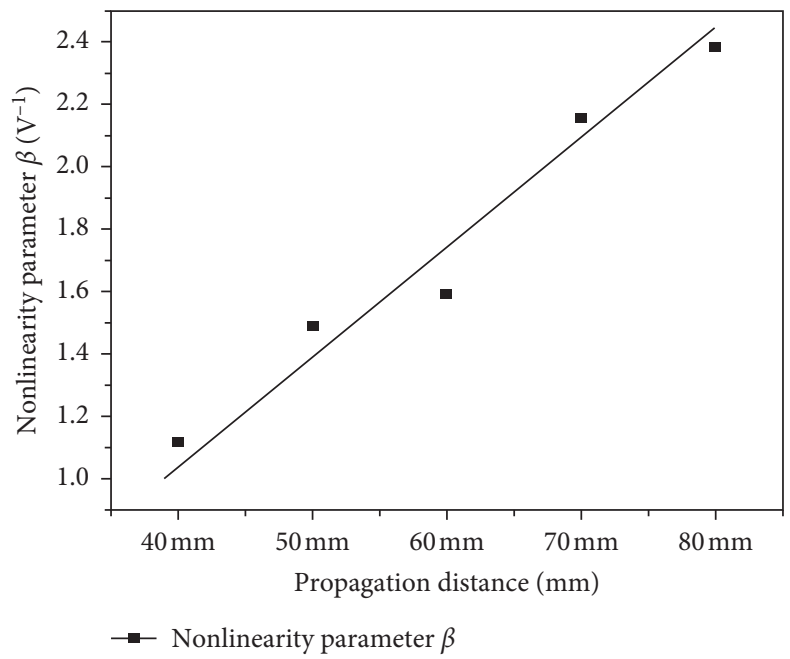

FIgURE 9: Relationship between nonlinear parameters and the propagation distance.

highly sensitive to the fatigue damage of high-strength FV520B steel. The relationship between the ultrasonic nonlinear parameters and fatigue cycles can be used to 


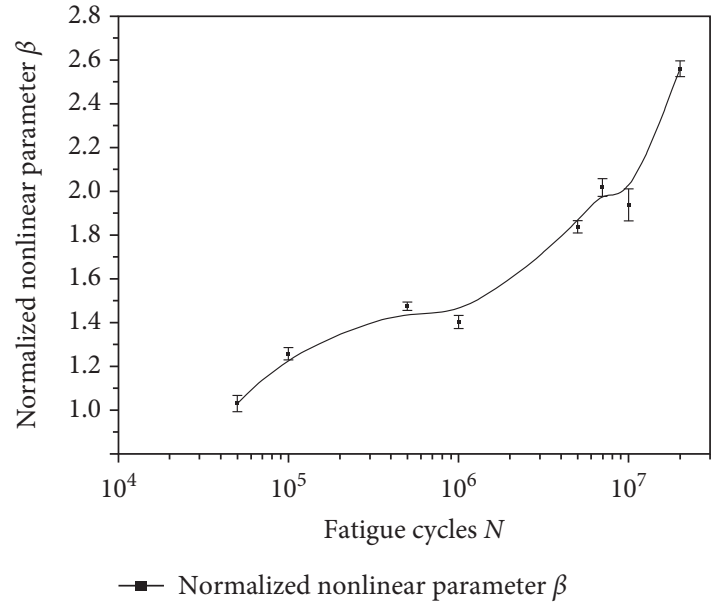

(a)

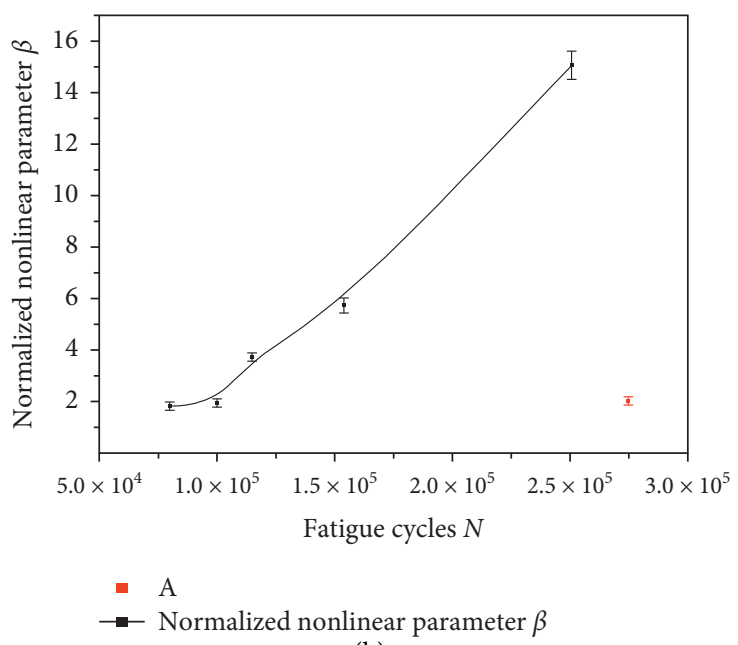

(b)

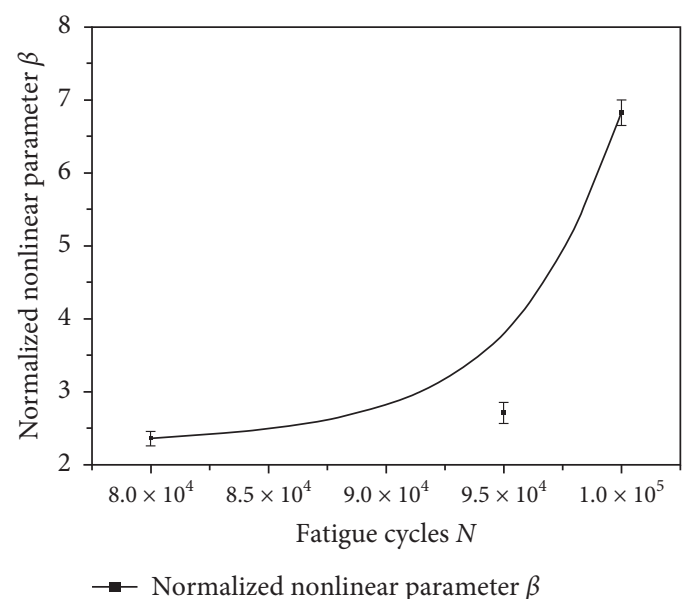

(c)

FIGURE 10: Relationship between nonlinearity parameter and fatigue cycles. (a) Relationship between nonlinearity parameter and fatigue cycles of plate specimen ( $400 \mathrm{MPa}$ loading stress). (b) Relationship between nonlinearity parameter and fatigue cycles of the notched specimen (550 MPa stress concentration). (c) Relationship between nonlinearity parameter and fatigue cycles of the notched specimen (660 MPa stress concentration).

characterize the early fatigue degree of the material. If the ultrasonic nonlinear parameters of specific parts of the material are calibrated in advance, it is expected that nonlinear ultrasonic nondestructive testing technology can be used to detect the fatigue degree of in-service parts on a regular basis.

\section{Microstructure Observation and Discussion}

5.1. Method and Sample for Microscopic Observation. The main methods of microstructure observation include optical microscopy and SEM. For the plate specimens, a cross section of the specimen was observed using SEM. The specimen was cut in the middle position. It was then inlaid, polished, and ultrasonically cleaned before being observed by SEM. The microscopic observation sample is shown in Figure 11. Zeiss field emission SEM was used to observe the samples. For the notched specimens, the growth of the main crack on the surface of the notch of the fatigue specimen was observed under the optical microscope, including the crack morphology and the length of the main crack. The notched sample was then cut off using an Instron universal material testing machine, and its section was observed under SEM. The purpose of the microscopic observation is to compare the microscopic damages of specimens with different fatigue degrees. Therefore, it is necessary to have the observation conditions as consistent as possible, to ensure the accuracy of comparison. The observation conditions include the observation area and magnification.

5.2. Microscopic Observations. The specimens of highstrength FV520B with different fatigue cycles were observed by the aforementioned experimental instruments and microscopic observation methods, while maintaining the same experimental conditions as long as possible.

Figure 12 shows the main crack morphology of the notched specimen, as observed under the metallographic 


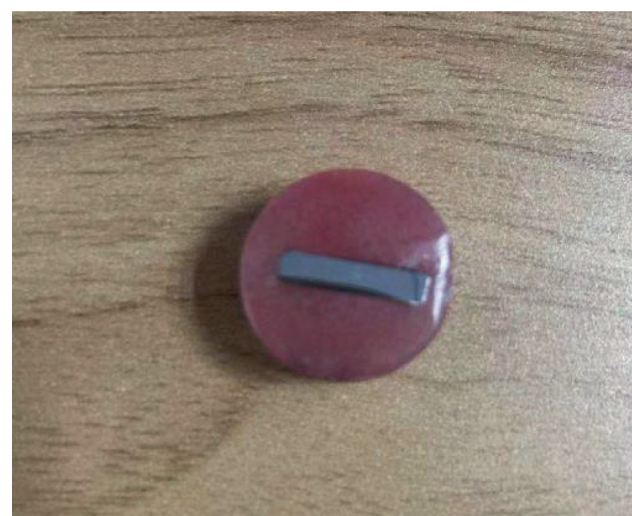

(a)

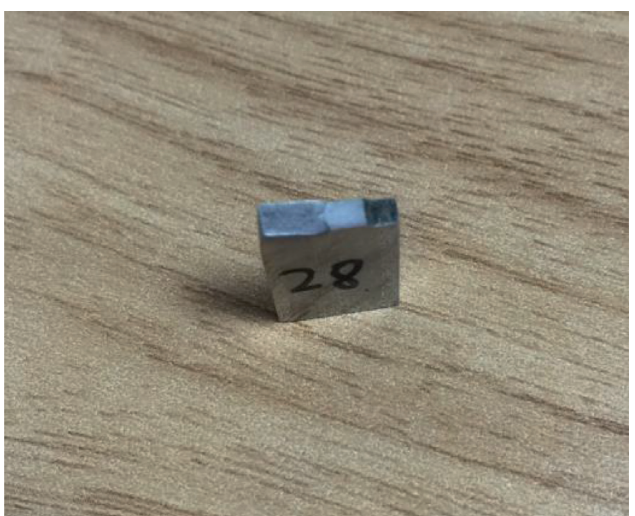

(b)

FIgURE 11: Microscopic observation sample. (a) Plate specimen. (b) Notched specimen.

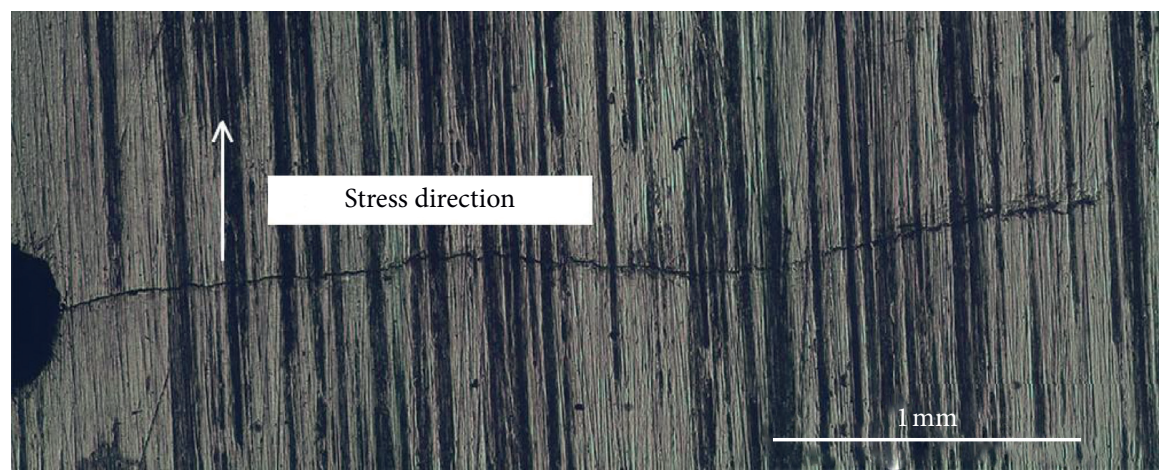

(a)

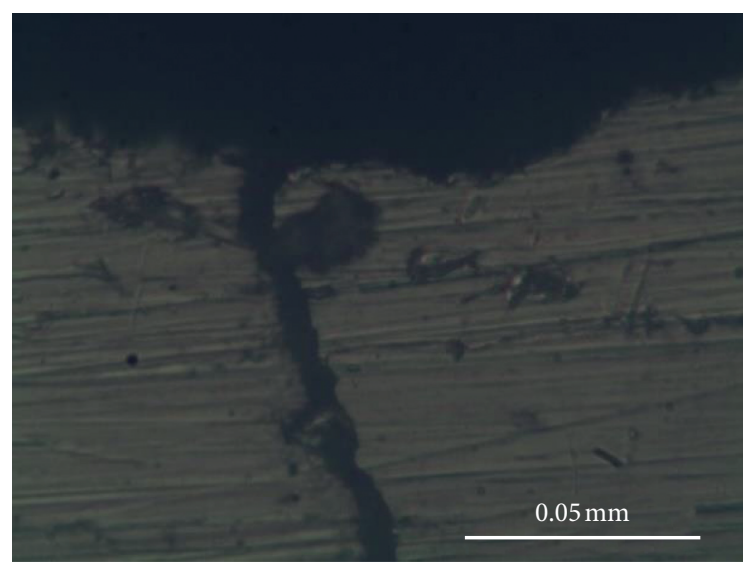

(b)

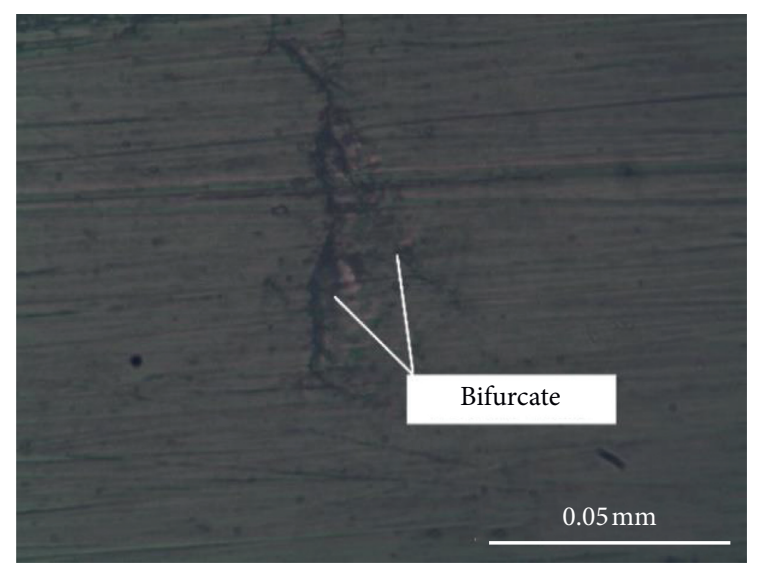

(c)

FIGURE 12: Morphology of the main crack in notched specimen. (a) Overall macroscopic appearance of the main crack. (b) Crack near the notch. (c) Crack tip.

microscope. The propagation path of the main crack is perpendicular to the loading direction, and the crack ends are bifurcated. Figure 13 presents the fracture morphology of the notched specimen. The fracture surfaces of the fatigue crack propagation regions of high-strength FV520B steel are flat. A large number of microcracks were found in the crack source region and fatigue growth region, and the surface morphology of tensile fracture regions has a dimpled shape. The boundary between the crack propagation regions and the tensile fracture regions has an arc shape.

The micrograph of the high-strength FV520B plate specimen (with $400 \mathrm{MPa}$ loading stress) under different fatigue cycles is shown in Figure 14. As shown, the 


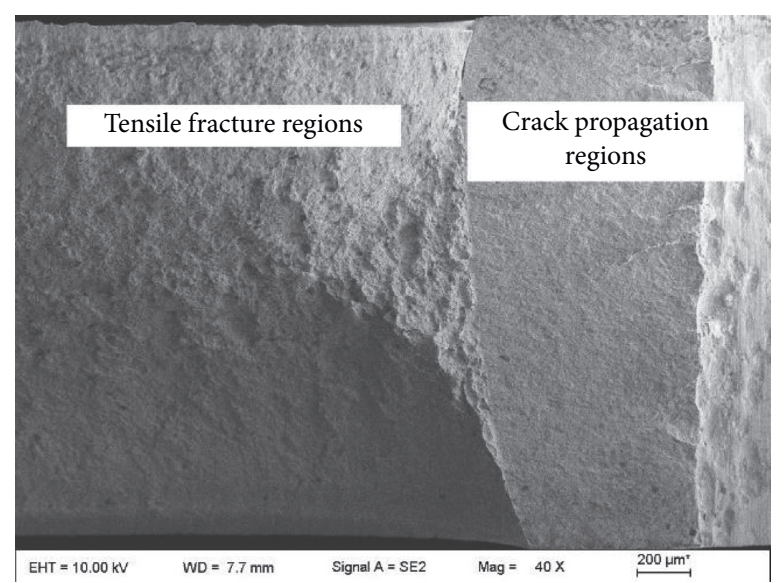

(a)

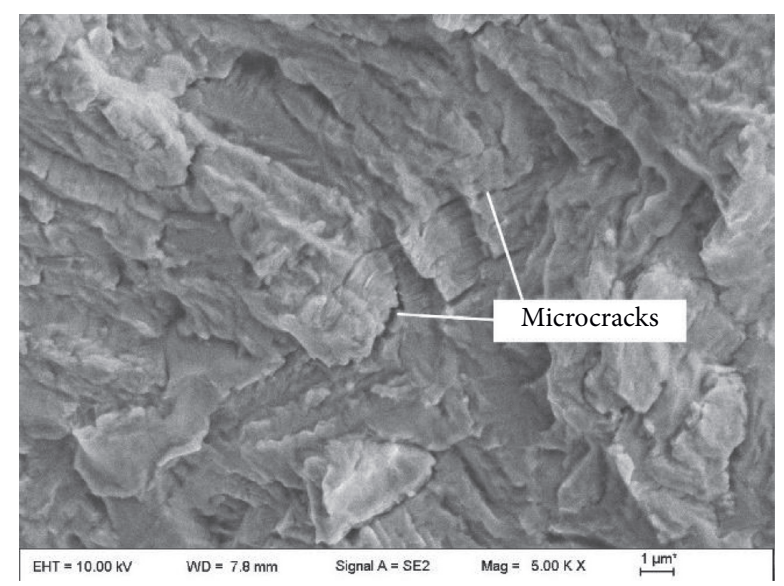

(b)

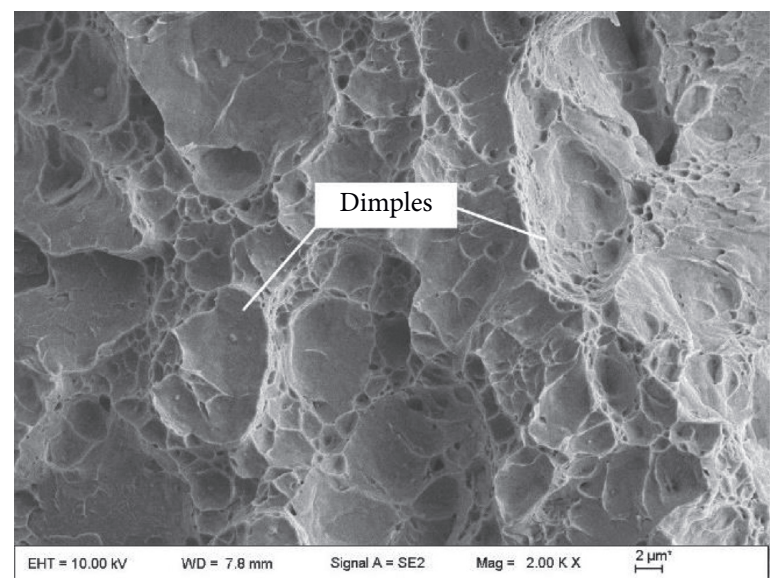

(c)

FIGURE 13: Fracture morphology of notched specimen. (a) Overall appearance of cross section. (b) Crack propagation regions. (c) Tensile fracture regions.

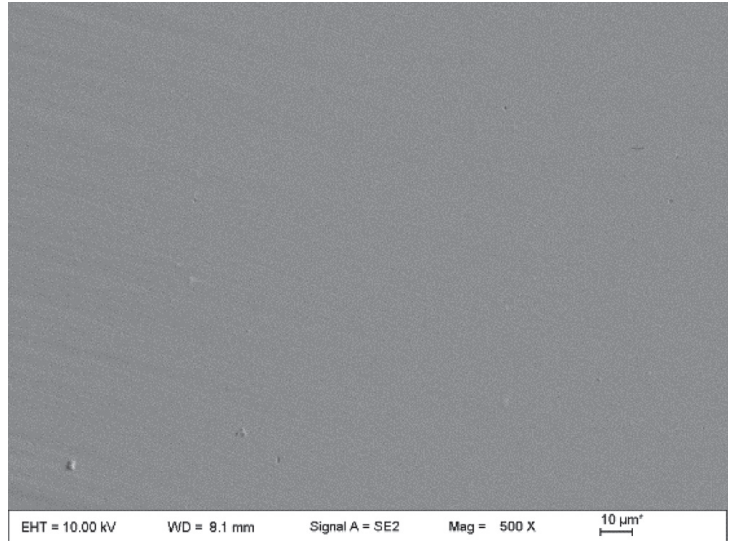

(a)

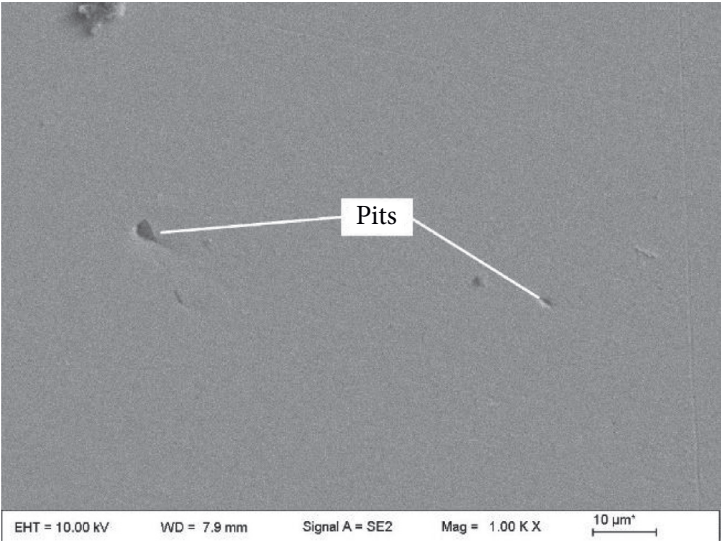

(b)

FIgUre 14: Continued. 


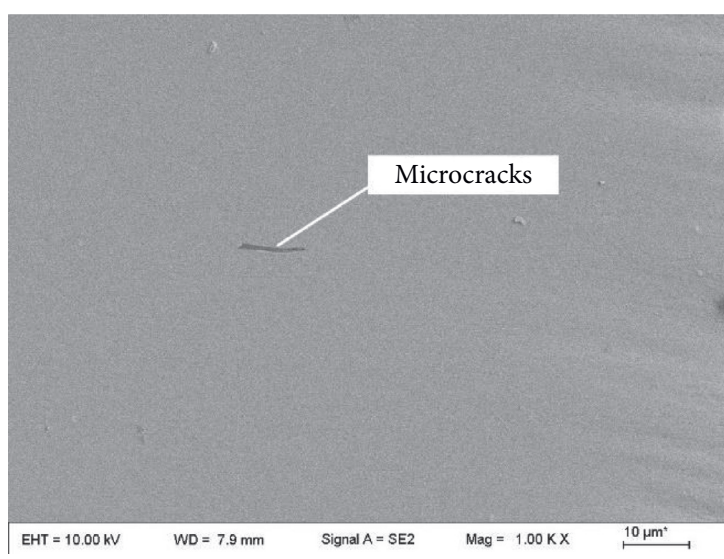

(c)

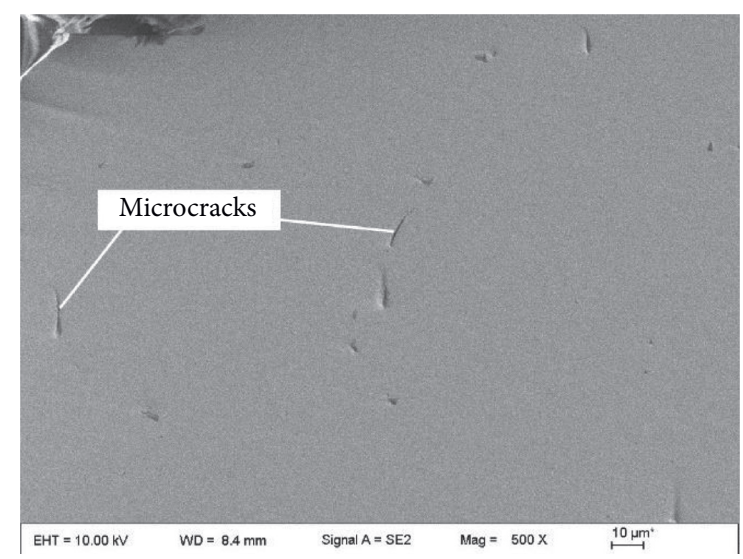

(d)

FIGURE 14: Microstructure of plate specimen (400 MPa loading stress). (a) Original microstructure. (b) Microstructure of $5 \times 10^{5}$ cycles. (c) Microstructure of $5 \times 10^{6}$ cycles. (d) Microstructure of $2 \times 10^{7}$ cycles.

microstructure of the material deteriorates with the increase of fatigue cycles. The matrix of the original specimen is relatively flat and has no evident defects, and its corresponding nonlinear parameters are relatively low. When the number of fatigue cycles reached $5 \times 10^{5}$, some small defects (such as pits) appeared in the material matrix, and the nonlinear parameters also increased. With further increase of fatigue cycles, the number of microholes increased significantly, microcracks began to appear, and the nonlinear parameters continued to increase. When the number of fatigue cycles increased to $2 \times 10^{7}$, the microcracks increased significantly, and the nonlinear parameters continued to increase. When the sinusoidal ultrasonic wave was transmitted into the solid medium, a nonlinear interaction occurred between the ultrasonic wave and the solid medium, leading to the generation of high-frequency harmonics. The generation of these harmonics is closely related to the nonlinearity of the microstructure of the solid media and is usually caused by internal defects of materials such as dislocations, micropores, and cracks [24-26]. In this experiment, with the increase in the number of fatigue cycles, the microstructures of the specimens gradually deteriorated, and the nonlinear parameters increased accordingly. Therefore, we can conclude that these deteriorating microstructures (as evidenced by defects such as micropores and cracks) lead to the generation of second harmonics. The results indicate that there is a certain correspondence between the nonlinear parameters and the internal damage of the material and that the nonlinear parameters can characterize the fatigue damage of highstrength FV520B. The micrographs of the FV520B notched specimens corresponding to $550 \mathrm{MPa}$ and $660 \mathrm{MPa}$ stress concentrations under different fatigue cycles are shown in Figures 15 and 16, respectively. As the specimen ultimately fractures, the fracture is divided into a fatigue source zone, fatigue crack expansion zone, and last tensile fracture zone. The variation of microcrack density can be obtained using the statistics of the microcracks. It can be used to verify the experimental results of the nonlinear ultrasound testing and to establish the relationship between the changes of the ultrasonic nonlinear parameters and the changes of the microstructure.

\subsection{Analysis of Microscopic Observations. For the plate} specimen (400 MPa loading stress), with the increase of the number of fatigue cycles, the microstructure of the specimen gradually deteriorated, as shown in Figure 14. For the experiments involving the two groups of notched specimens, the main crack propagated in the notch with an increase of fatigue cycles, as the specimen was in a state of stress concentration in the notch. The morphology and size of the main crack were measured using a metallographic microscope. In addition, with the increase of fatigue cycles, the number and sizes of the microcracks increased, eventually leading to the failure of the materials. The process of increasing the fatigue cycle is associated with fatigue microcrack initiation and propagation. Therefore, we can observe the fracture surface of the specimen and calculate its microcrack distribution.

For this experiment, it was necessary to count the crack distributions of notched specimens with different fatigue cycles. This process can be carried out in two steps. First, it is necessary to select an appropriate and identical observation area on the micrograph of each specimen. As the crack distributions in the micrographs of each specimen are not absolutely uniform, an area with clear cracks and uniform crack distribution should be selected as the statistical area (as best as possible). Second, we calculate the number and length of microcracks in the statistical region of the micrograph of each specimen. As the sizes of the microcracks in the statistical area of the same micrograph can be different, we should select clear and complete microcracks when counting the number of microcracks. Moreover, as the statistical area of each image is the same, an equivalent microcrack length (i.e., the sum of microcrack lengths) can be used to directly represent the changes in microcrack density. The statistical results of the cracks in the notched specimens are shown in Tables 3 and 4, respectively. Figure 17 shows the relationships between the main crack 


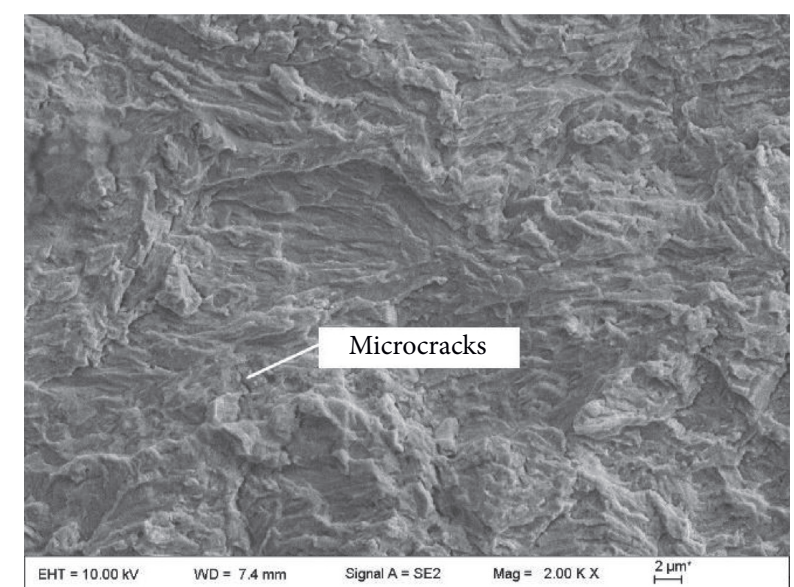

(a)

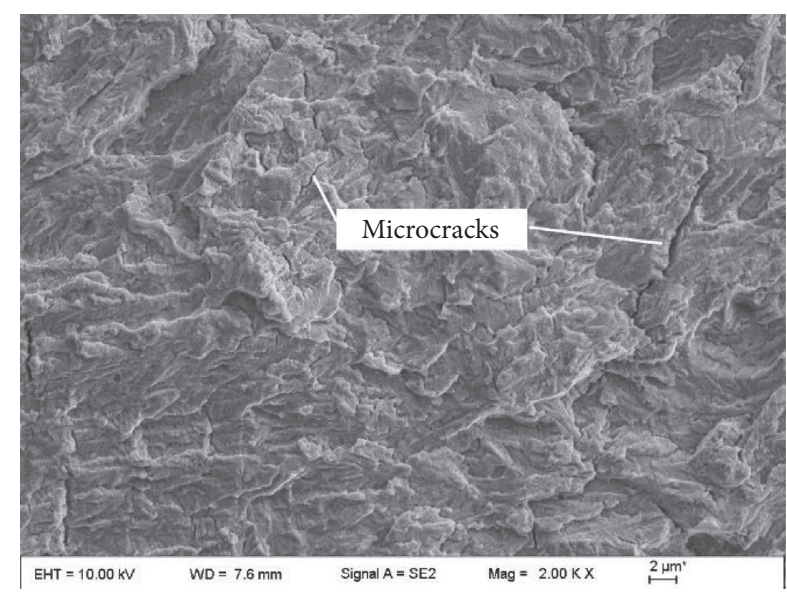

(b)

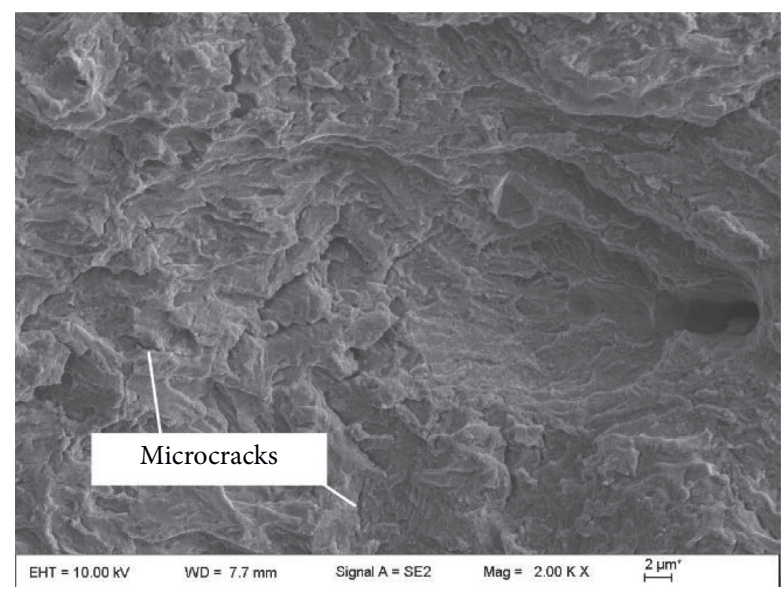

(c)

FIGURE 15: Microstructure of notched specimens (550 MPa stress concentration). (a) Microstructure of $1.00 \times 10^{5}$ cycles. (b) Microstructure of $2.50 \times 10^{5}$ cycles. (c) Microstructure of $2.75 \times 10^{5}$ cycles.

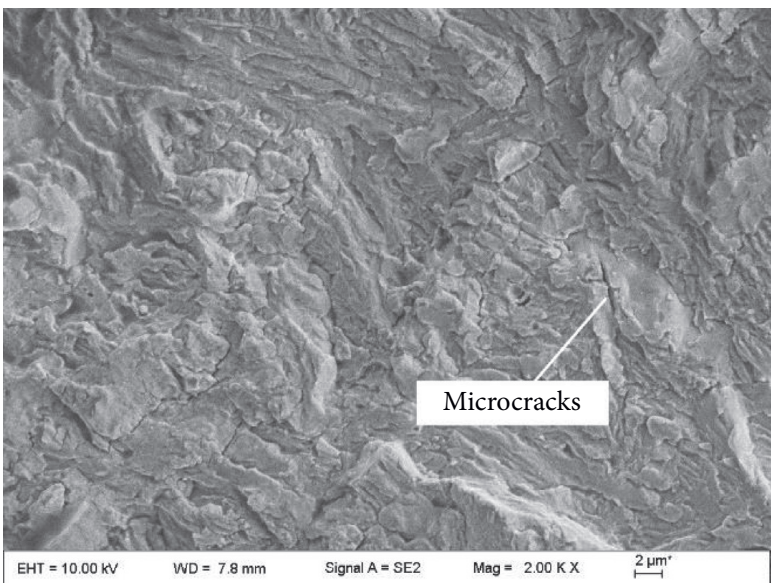

(a)

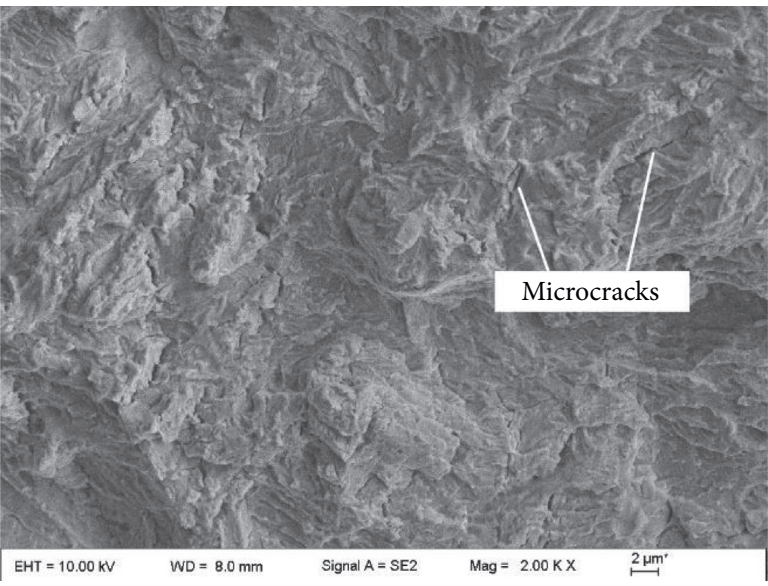

(b)

FIgURE 16: Continued. 


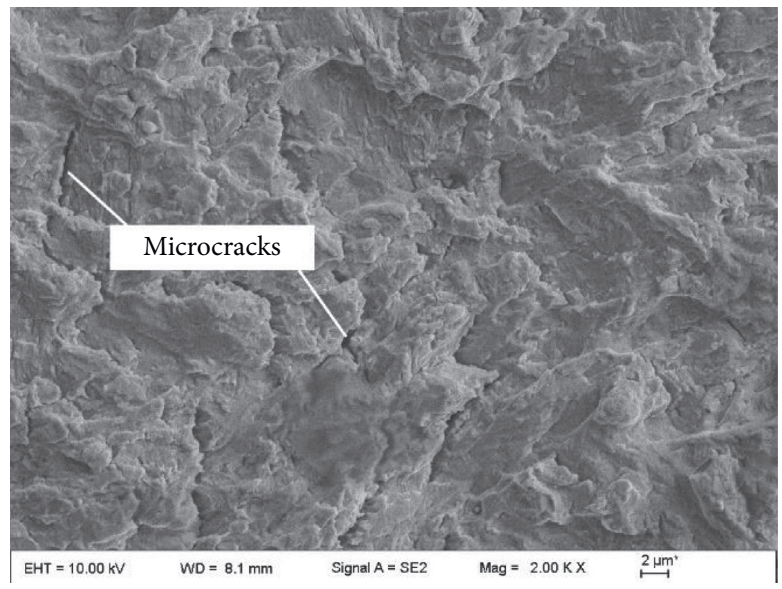

(c)

FiguRe 16: Microstructure of notched specimens ( $660 \mathrm{MPa}$ stress concentration). (a) Microstructure of $8.00 \times 10^{4}$ cycles. (b) Microstructure of $9.50 \times 10^{4}$ cycles. (c) Microstructure of $1.00 \times 10^{5}$ cycles.

TABLE 3: Statistics of the cracks in the notched specimens (550 MPa stress concentration).

\begin{tabular}{lccc}
\hline Samples number & Sample no. 1 & Sample no. 2 & Sample no. 3 \\
\hline Main crack length $(\mathrm{mm})$ & 1.03 & 3.10 & 6.56 \\
Number of microcracks & 25 & 10 & 29 \\
Length of largest microcrack $(\mu \mathrm{m})$ & 10.993 & 7.993 & 14.883 \\
Equivalent microcrack length $(\mu \mathrm{m})$ & 142.503 & 60.268 & 185.58 \\
\hline
\end{tabular}

TABLE 4: Statistics of the cracks in the notched specimens (660 MPa stress concentration).

\begin{tabular}{lccc}
\hline Samples number & Sample no. 4 & Sample no. 5 & Sample no. 6 \\
\hline Main crack length $(\mathrm{mm})$ & 0.64 & 1.01 & 3.04 \\
Number of microcracks & 20 & 24 & 32 \\
Length of largest microcrack $(\mu \mathrm{m})$ & 9.541 & 10.622 & 16.63 \\
Equivalent microcrack length $(\mu \mathrm{m})$ & 105.412 & 107.967 & 262.814 \\
\hline
\end{tabular}

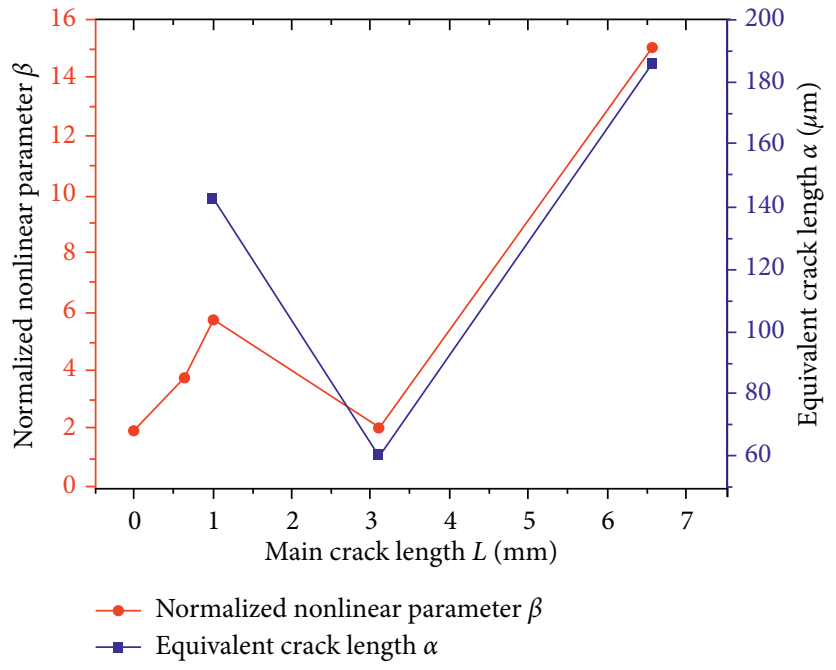

FiguRE 17: Relationship between equivalent microcrack length, ultrasonic nonlinear parameters, and main crack length of notched specimen (550 MPa stress concentration). 


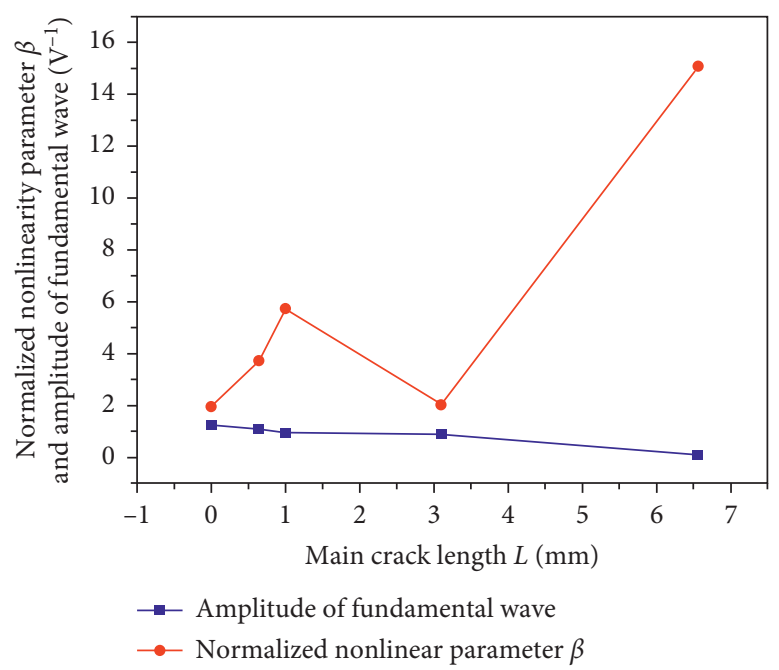

FIGURE 18: Ultrasonic nonlinear parameters of the notched specimen (550 MPa stress concentration) with different main crack lengths.

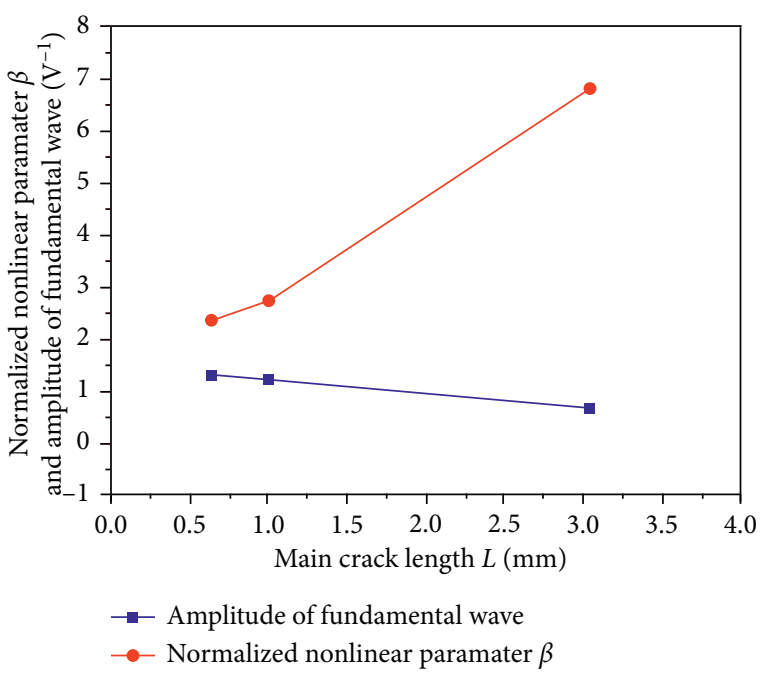

FIGURE 19: Ultrasonic nonlinear parameters of the notched specimen (660 MPa stress concentration) with different main crack lengths.

length and the equivalent microcrack length and ultrasonic nonlinear parameters, respectively.

As shown in Figure 18, when the length of the main crack is less than $3 \mathrm{~mm}$, the amplitude of the fundamental wave changes slightly. In contrast, the ultrasonic nonlinear parameters change significantly. The equivalent microcrack length of the specimen cross section was calculated, and it was found that the equivalent microcrack length with the ultrasonic nonlinear parameters had better consistency than the main crack length, as shown in Figure 17. The ultrasonic nonlinear parameters increase with the increase of the length of the main crack, but not monotonically. When the length of the main crack reaches $3.1 \mathrm{~mm}$ (corresponding to point $\mathrm{A}$ in Figure 10(b)), the ultrasonic nonlinear parameters evidently decrease, and the equivalent length of the microcrack also shows corresponding changes. This further indicates that the ultrasonic nonlinear effect is related to the

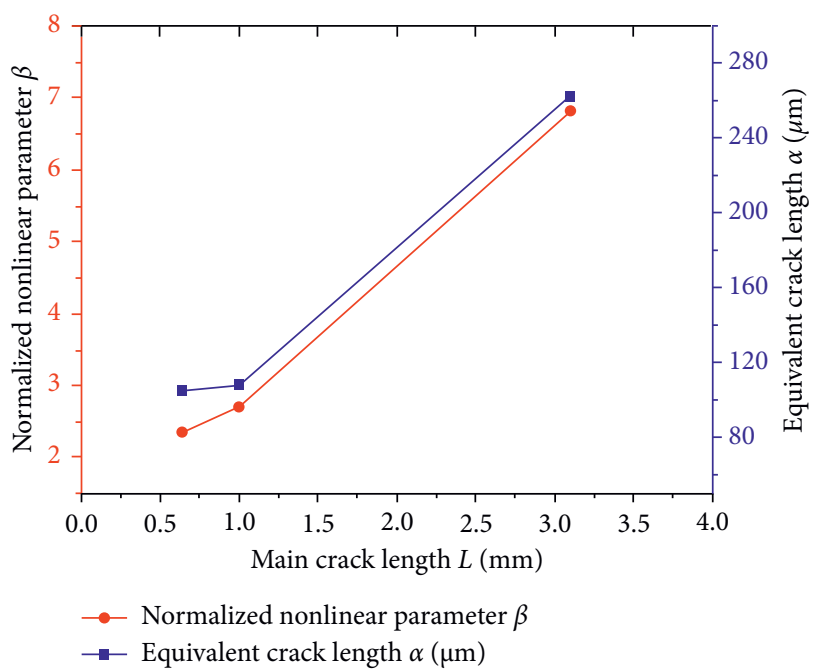

FIGURE 20: Relationship between equivalent microcrack length, ultrasonic nonlinear parameters, and main crack length of notched specimen (660 MPa stress concentration).

equivalent microcrack length in the specimen. The ultrasonic nonlinear parameters can well characterize the changes of microcracks in high-strength FV520B and indicate the fatigue damage degree of the material. Similar results were obtained in the notched specimen $(660 \mathrm{MPa}$ stress concentration) experiment. As shown in Figures 19 and 20, with the increase of the main crack size, the ultrasonic nonlinear parameters were more sensitive than the fundamental amplitude. The variation trends of the equivalent microcrack length and ultrasonic nonlinear parameters have better consistency.

\section{Conclusions}

Nonlinear ultrasonic tests were performed on two types of fatigue specimens (plate specimens and notched specimens), and the $\beta-N$ curves of FV520B under three stress levels were 
obtained. The results show that the ultrasound nonlinear parameter is highly sensitive to the early fatigue damage of the material.

The microstructure was observed using SEM. The results indicate that the change of ultrasonic nonlinear parameters is related to the deterioration of the microstructure of the material. The nonlinear parameters can characterize the fatigue damage of FV520B material.

The relationship between the ultrasonic nonlinear parameters and the length of the main crack and equivalent microcrack length is analyzed. As compared with the length of the main crack, the equivalent microcrack length is more consistent with the ultrasonic nonlinear parameters, indicating that the nonlinear parameters are mainly due to the appearance of the internal microcrack.

\section{Data Availability}

The data used to support the findings of this study are included within the article.

\section{Conflicts of Interest}

The authors declare no conflicts of interest.

\section{Authors' Contributions}

B.C. conceptualized the study, contributed to formal analysis and resources, and was responsible for funding acquisition; C.W. contributed to methodology, performed data curation, and prepared the original draft; C.W. and P.W. validated the study; P.W. reviewed and edited the manuscript; S.Z. performed study supervision; W.S. was involved in project administration.

\section{Acknowledgments}

This study was supported by the National Natural Science Foundation of China (no. 51905484) (Research on very high cycle fatigue damage evaluation and life estimation method of centrifugal compressor impeller based on nonlinear ultrasonic testing). The paper was edited by Elsevier Language Editing Services.

\section{References}

[1] W. Q. He, "Full-life mechanical response analysis of large centrifugal compressor impeller," Master thesis, Dalian University Of Technology, Dalian, 2010.

[2] L. S. Shu, "Research on service life prediction model and numerical simulation of centrifugal compressor remanufactured impeller," Doctoral Dissertation, Chongqing University, Chongqing, China, 2013.

[3] M. Zhang, "Study on ultra high cycle fatigue behavior and mechanism of FV520B centrifugal compressor impeller material," Doctoral dissertation, Shandong University, Jinan, China, 2015.

[4] C. W. Wu, Z. Q. Guan, X. L. Guo et al., "Fatigue reliability analysis of large centrifugal compressor impeller blades," Equipment Manufacturing Technology, vol. 8, pp. 1-3, 2008.
[5] Y. Meng, L. Li, and Q. H. Li, "Transient analysis method of blade forced response under wake excitation," Journal of Beijing University of Aeronautics and Astronautics, vol. 32, pp. 671-674, 2006.

[6] J. H. Cantrell, "Substructural organization, dislocation plasticity and harmonic generation in cyclically stressed wavy slip metals," Proceedings of the Royal Society of London. Series A: Mathematical, Physical and Engineering Sciences, vol. 460, no. 2043, pp. 757-780, 2004.

[7] G. Shui, J.-Y. Kim, J. Qu, Y.-S. Wang, and L. J. Jacobs, “A new technique for measuring the acoustic nonlinearity of materials using Rayleigh waves," NDT \& E International, vol. 41, no. 5, pp. 326-329, 2008.

[8] K. Jhang and K. Kim, "Evaluation of material degradation using nonlinear acoustic effect," Ultrasonics, vol. 37, pp. 3944, 1997.

[9] M. X. Deng and J. F. Pei, "Nonlinear ultrasonic Lamb wave response to fatigue of solid plates," Acta Acoustics, vol. 33, pp. 360-369, 2008.

[10] S. V. Walker, J. Y. Kim, J. Qu, and L. J. Jacobs, "Fatigue damage evaluation in A36 steel using nonlinear Rayleigh surface waves," NDT \& E International : Independent Nondestructive Testing and Evaluation, vol. 48, pp. 10-15, 2012.

[11] J. F. Zhang, "Study on nonlinear ultrasonic detection and evaluation of austenitic stainless steel service damage," Doctoral Dissertation, East China University of Science and Technology, Shanghai, China, 2014.

[12] Z. Wang, P. Qiao, and B. Shi, "Nonpenetrating damage identification using hybrid lamb wave modes from hilberthuang spectrum in thin-walled structures," Shock and Vibration, vol. 2017, Article ID 5164594, 11 pages, 2017.

[13] D. Dutta, H. Sohn, K. A. Harries, and P. Rizzo, "A nonlinear acoustic technique for crack detection in metallic structures," Structural Health Monitoring: An International Journal, vol. 8, no. 3, pp. 251-262, 2009.

[14] Y. Shen, J. Wang, and W. Xu, "Nonlinear features of guided wave scattering from rivet hole nucleated fatigue cracks considering the rough contact surface condition," Smart Materials and Structures, vol. 27, no. 10, p. 105044, 2018.

[15] Y. Shen and C. E. S. Cesnik, "Nonlinear scattering and mode conversion of Lamb waves at breathing cracks: an efficient numerical approach," Ultrasonics, vol. 94, pp. 202-217, 2019.

[16] Y. Shen and C. E. S. Cesnik, "Modeling of nonlinear interactions between guided waves and fatigue cracks using local interaction simulation approach," Ultrasonics, vol. 74, pp. 106-123, 2017.

[17] M. Hong, Z. Su, Q. Wang, L. Cheng, and X. Qing, "Modeling nonlinearities of ultrasonic waves for fatigue damage characterization: theory, simulation, and experimental validation," Ultrasonics, vol. 54, no. 3, pp. 770-778, 2014.

[18] X. Liu, L. Bo, Y. Liu et al., "Detection of micro-cracks using nonlinear lamb waves based on the Duffing-Holmes system," Journal of Sound and Vibration, vol. 405, pp. 175-186, 2017.

[19] Q. Wu, R. Wang, F. Yu, and Y. Okabe, "Application of an optical fiber sensor for nonlinear ultrasonic evaluation of fatigue crack," IEEE Sensors Journal, vol. 19, no. 13, pp. 4992-4999, 2019.

[20] R. Wang, Q. Wu, F. Yu, Y. Okabe, and K. Xiong, "Nonlinear ultrasonic detection for evaluating fatigue crack in metal plate," Structural Health Monitoring, vol. 18, no. 3, pp. 869-881, 2019.

[21] K.-Y. Jhang, "Nonlinear ultrasonic techniques for nondestructive assessment of micro damage in material: a review," International Journal of Precision Engineering and Manufacturing, vol. 10, no. 1, pp. 123-135, 2009. 
[22] Y. X. Xiang, M. X. Deng, and F. Z. Xuan, "Creep damage characterization using nonlinear ultrasonic guided wave method: a mesoscale model," Journal of Applied Physics, vol. 115, p. 044914, 2014.

[23] Y. Xiang, W. Zhu, C.-J. Liu, F.-Z. Xuan, Y.-N. Wang, and W.-C. Kuang, "Creep degradation characterization of titanium alloy using nonlinear ultrasonic technique," $N D T \& E$ International, vol. 72, pp. 41-49, 2015.

[24] J. Herrmann, J.-Y. Kim, L. J. Jacobs, J. Qu, J. W. Littles, and M. F. Savage, "Assessment of material damage in a nickel-base superalloy using nonlinear Rayleigh surface waves," Journal of Applied Physics, vol. 99, no. 12, p. 124913, 2006.

[25] J.-Y. Kim, L. J. Jacobs, J. Qu, and J. W. Littles, "Experimental characterization of fatigue damage in a nickel-base superalloy using nonlinear ultrasonic waves," The Journal of the Acoustical Society of America, vol. 120, no. 3, pp. 1266-1273, 2006.

[26] W. Li, H. Cui, W. Wen, X. Su, and C. C. Engler-Pinto, "In situ nonlinear ultrasonic for very high cycle fatigue damage characterization of a cast aluminum alloy," Materials Science and Engineering: A, vol. 645, pp. 248-254, 2015. 